\title{
Triglyceride enrichment of HDL enhances in vivo metabolic clearance of HDL apo A-I in healthy men
}

\author{
Benoît Lamarche, ${ }^{1}$ Kristine D. Uffelman, ${ }^{1}$ André Carpentier, ${ }^{1}$ Jeffrey S. Cohn, ${ }^{2}$ \\ George Steiner, ${ }^{1}$ P. Hugh Barrett, ${ }^{3}$ and Gary F. Lewis ${ }^{1}$ \\ ${ }^{1}$ Department of Medicine, Division of Endocrinology, University of Toronto, Toronto, Ontario, Canada MSG 2C4
${ }^{2}$ Clinical Research Institute of Montreal, Montreal, Quebec, Canada H2W 1R7
${ }^{3}$ The Resource Facility for Population Kinetics, Department of Bioengineering, University of Washington, Seattle,
Washington 98195, USA
}

Address correspondence to: Gary Lewis, The Toronto Hospital, General Division, 200 Elizabeth Street, Room EN11-229, Toronto, Ontario, Canada M5G 2C4. Phone: (416) 340-4270; Fax: (416) 340-3314; E-mail: glewis@torhosp.toronto.on.ca

Received for publication September 21, 1998, and accepted in revised form March 12, 1999.

\begin{abstract}
Triglyceride (TG) enrichment of HDL resulting from cholesteryl ester transfer protein-mediated exchange with TG-rich lipoproteins may enhance the lipolytic transformation and subsequent metabolic clearance of HDL particles in hypertriglyceridemic states. The present study investigates the effect of TG enrichment of HDL on the clearance of HDL-associated apo A-I in humans. HDL was isolated from plasma of six normolipidemic men (mean age: $29.7 \pm 2.7$ years) in the fasting state and after a fivehour intravenous infusion with a synthetic TG emulsion, Intralipid. Intralipid infusion resulted in a 2.1-fold increase in the TG content of HDL. Each tracer was then whole-labeled with ${ }^{125} \mathrm{I}$ or ${ }^{131} \mathrm{I}$ and injected intravenously into the subject. Apo A-I in TG-enriched HDL was cleared $26 \%$ more rapidly than apo A-I in fasting HDL. A strong correlation between the Intralipid-induced increase in the TG content of HDL and the increase in HDL apo A-I fractional catabolic rate reinforced the importance of TG enrichment of HDL in enhancing its metabolic clearance. HDL was separated further into lipoproteins containing apo A-II (LpAI:AII) and those without apo A-II (LpAI). Results revealed that the enhanced clearance of apo A-I from TG-enriched HDL could be largely attributed to differences in the clearance of LPAI but not LPAI:AII. This is, to our knowledge, the first direct demonstration in humans that TG enrichment of HDL enhances the clearance of HDL apo A-I from the circulation. This phenomenon could provide an important mechanism explaining how HDL apo A-I and HDL cholesterol are lowered in hypertriglyceridemic states.
\end{abstract}

J. Clin. Invest. 103:1191-1199 (1999).

\section{Introduction}

The inverse relationship between plasma HDL cholesterol concentrations and the risk of cardiovascular disease is well accepted $(1,2)$. There is a large body of evidence indicating that variations in plasma HDL cholesterol concentrations are inversely related to plasma triglyceride (TG) levels $(3,4)$. Hence, one of the most frequent metabolic abnormalities accompanying reduced plasma HDL cholesterol levels is hypertriglyceridemia. Apo A-I, the major protein of HDL, is a crucial structural and functional component in the metabolism of these particles. Studies have shown that the fractional catabolic rate (FCR) of apo A-I is a significant and powerful predictor of plasma HDL cholesterol levels (5, 6). Studies that have examined the production and clearance rates of apo A-I as a marker of HDL metabolism in humans have led to the hypothesis that hypertriglyceridemia may be one of several factors ultimately affecting plasma HDL cholesterol levels (7-9). However, these studies have not tested this hypothesis directly because they relied on correlations between HDL apo A-I FCR and plasma TG concentrations.

The mechanisms underlying the enhanced catabolism of apo A-I in hypertriglyceridemic states are not well understood. Hypertriglyceridemia is associated with an increased cholesteryl ester transfer protein-mediated (CETP-mediated) transfer of TG from the expanded pool of TG-rich lipoproteins to HDL and of cholesteryl ester from HDL to TG-rich lipoproteins (10). The resulting TG enrichment of the HDL particle makes it a better substrate for lipolysis by hepatic lipase, an enzyme that plays a key role in HDL metabolism $(11,12)$. Results from an ex vivo kidney perfusion study have indicated that TG enrichment of HDL alone, in the absence of subsequent lipolytic modification of the particle by hepatic lipase and lipoprotein lipase, may have very little impact, if any, on the uptake of apo A-I by the kidney (13). On the other hand, lipolytic modification of TG-rich HDL by lipoprotein lipase and hepatic lipase was associated with a significant increase in the uptake of apo A-I by the perfused rabbit kidney and loss of apo A-I from the HDL fraction (13). In vitro incubation of TG-enriched human HDL with hepatic lipase has also been shown to promote the loss of apo A-I from the particle (14). We have recently shown, using a rabbit model, that the FCR of apo A-I from small, lipolytically modified HDL was increased significantly compared with the FCR of apo A-I from large, TG-enriched HDL (15). This increase in HDL FCR was not observed in the absence of lipolytic modification of TG-rich HDL particles (16). In vitro and in vivo data sug- 
gest that a significant proportion of apo A-I on HDL may be in a readily dissociable form and that hypertriglyceridemia may augment the proportion of this dissociable pool of apo A-I. Horowitz et al. (13) have shown that individuals with reduced HDL cholesterol levels have more loosely bound, easily exchanged apo A-I and that this exchangeable pool of apo A-I may be cleared rapidly from the circulation. The recognition of the importance of TG enrichment of HDL as an important process regulating its catabolism comes also from several other indirect lines of evidence. These include studies in transgenic mice coexpressing human apo C-III (which causes hypertriglyceridemia), human apo A-I and CETP (17), or CETP alone (18); in vitro studies (19); and studies in monkeys infused with an anti-lipoprotein lipase antibody (20).

Although in vitro and ex vivo studies in both animals and humans have provided indirect evidence that TG enrichment of HDL may have a significant impact in determining the rate at which apo A-I is catabolized, there are no studies to date that have directly tested this hypothesis in humans. The present study was therefore undertaken to investigate the effects of TG enrichment of HDL as one of the potential mechanisms leading to an increased metabolic clearance of apo A-I frequently seen in hypertriglyceridemic states.

\section{Methods}

Subjects. Twelve healthy, normolipidemic men participated in the study. A first sample of six men aged $22-38$ years with a mean $\left( \pm\right.$ SEM) body mass index of $24.4 \pm 1.2 \mathrm{~kg} / \mathrm{m}^{2}$ participated in the kinetic study. Their lipid profile is presented in Table 1. A second sample of six men of similar age, body weight, and lipid profile (cholesterol: $4.0 \pm 0.6 \mathrm{mmol} / \mathrm{l}$; TG: $0.98 \pm 0.29$ $\mathrm{mmol} / \mathrm{l}$; HDL cholesterol: $1.04 \pm 0.31 \mathrm{mmol} / \mathrm{l}$ ) were recruited to characterize further the effects of changes induced by Intralipid (Baxter Corp., Mississauga, Ontario, Canada) in the phospholipid and apolipoprotein content of HDL (see later here). This latter group did not undergo an HDL kinetic study. All participants were nonsmokers and did not have a previous history of cardiovascular disease or any other systemic illness. None of the participants were using any pharmacologic agents. All subjects gave their written informed consent to participate in the study, which was approved by the Human Ethics Committee of The Toronto Hospital, University of Toronto.

The study protocol comprised two phases. In phase 1, HDL from fasting plasma and from plasma that had been enriched with TG by a five-hour intravenous infusion of Intralipid was isolated and radiolabeled. In the second phase of the study, the two autologous HDL tracers were injected into the participants, and the clearance of apo A-I associated with each tracer was determined over a five-day period.

Phase 1 isolation and iodination of fasting and TG-enriched HDL. Before their first visit to the Metabolic Investigation Unit of The Toronto Hospital, all participants were instructed to maintain their usual diet and to refrain from consuming alcohol for at least three days prior to the start of the study. Two intravenous lines were inserted into forearm veins at 8:00 am, one in each arm, following a 14-h overnight fast; one line was for blood sampling and the other for infusion of Intralipid. Intralipid is a sterile fat emulsion containing $20 \%$ soybean oil, $1.2 \%$ egg phospholipids, and $2.25 \%$ glycerin in water. Approximately $100 \mathrm{ml}$ of blood was first drawn under sterile conditions into Vacutainer tubes Becton Dickinson Vacutain Systems (Rutherford, New Jersey, USA) containing $1.2 \mathrm{~g} / \mathrm{l}$ sodium EDTA for the isolation of fasting HDL, as described later here. Intralipid was then infused intravenously for five hours $(50 \mathrm{cc} / \mathrm{h})$, with the subject resting in the recumbent position. Subjects were not permitted to eat until completion of the Intralipid infusion. After five hours, a second blood sample of $100 \mathrm{ml}$ was drawn into sterile EDTA-containing tubes for the subsequent isolation of TG-enriched HDL. Preliminary studies showed that total plasma TG and HDL TG levels increased and then tended to reach a plateau at approximately five hours in normolipidemic subjects (results not shown).

Fasting and TG-enriched HDL were isolated by sequential ultracentrifugation of whole plasma at consecutive densities $(d)$ of $1.063 \mathrm{~g} / \mathrm{ml}$ for $20 \mathrm{~h}$ at $40,000 \mathrm{~g}$ and $1.21 \mathrm{~g} / \mathrm{ml}$ for $27 \mathrm{~h}$ at $60,000 \mathrm{~g}$ at $4^{\circ} \mathrm{C}$ in a Beckman 70.1 Ti rotor (Beckmen Instruments Inc., Misisauga, Ontario, Canada) and then dialyzed overnight in a buffer containing $0.15 \mathrm{M} \mathrm{NaCl}, 0.01 \mathrm{M}$ Tris-base, and $5 \mathrm{mM}$ EDTA (pH 8.0). As described previously (16), both tracers were whole-labeled with $1 \mathrm{mCi}$ of either ${ }^{125} \mathrm{I}$ or ${ }^{131} \mathrm{I}$, according to a modification of the iodine monochloride method of McFarlane (21). The ${ }^{125} \mathrm{I}$ and ${ }^{131}$ I radioactive labels were alternated between fasting and TG-enriched HDL in each experiment, to prevent a systematic "isotope effect" on the clearance of the tracers. Thus, TG-enriched HDL was labeled with ${ }^{131} \mathrm{I}$ in three experiments and with ${ }^{125} \mathrm{I}$ in three experiments. Approximately 2-3 mg of fasting and TG-enriched HDL protein was used for the iodination protocol. An equal volume of unlabeled TG-enriched and fasting HDL was added to the radioiodinated HDL fractions as a cold carrier, and HDL tracers were washed at $d=1.21 \mathrm{~g} / \mathrm{ml}, 39,000 \mathrm{~g}, 4^{\circ} \mathrm{C}$ in a 70.1 Ti rotor for $40 \mathrm{~h}$, followed by dialysis in Tris buffer at $4^{\circ} \mathrm{C}$. The composition of each radiolabeled tracer was measured using commercially available enzymatic assay kits as described here. Analysis of composition and size by $4-30 \%$ nondenaturing PAGE of the fasting and TG-enriched HDL before and after radioiodination indicated that the iodination process had no significant effect on these parameters (results not shown). We also determined the amount of radioactivity in the $d>1.21$ $\mathrm{g} / \mathrm{ml}$ bottom fraction in three subjects after isolating and washing the HDL tracers, and we found no difference in the loss from fasting and TG-rich HDL fractions. Each tracer was tested for pyrogenicity and sterility before reinjection into the human subject. The length of time between isolation and reinjection of the HDL tracers was one week.

Phase 2 HDL turnover study. Three days before the beginning of the HDL turnover study, the study participants were provided with and instructed to eat only an American Heart Association (AHA) phase 1 isocaloric diet, consisting of approximately $50 \%$ carbohydrate, $30 \%$ fat, and $20 \%$ protein. Subjects were firmly instructed to refrain from consuming any form of alcohol during the course of the turnover study. All participants were also instructed to take potassium iodide at a dose of $300 \mathrm{mg} / \mathrm{day}$ for a total of five weeks, beginning three days before the administration of the radiolabeled HDL, to prevent thyroidal and salivary gland accumulation of radioiodide. After a 12-h overnight fast, $50 \mu \mathrm{Ci}$ of ${ }^{125} \mathrm{I}-\mathrm{HDL}$ and $100 \mu \mathrm{Ci}$ of ${ }^{131} \mathrm{I}$-HDL were injected simultaneously into a forearm vein of the subject. Blood samples $(20 \mathrm{ml})$ were obtained over the next five days at the following time intervals: 10 and $30 \mathrm{~min}$, and 1, 2, 4, 6, 10, 17, 24, 32, $48,56,72$, and $96 \mathrm{~h}$. Subjects remained in the fasting state for the initial six hours, after which they were provided with a light snack consisting of low-fat foods such as bread, slow-release carbohydrate energy bars, fruits, and vegetables. The fat content of these foods was less than $10 \%$ to avoid major excursions in postprandial TG-rich lipoproteins. After the 17-h blood sample, participants were given a regular meal and then fasted until the next morning (24-h blood sample). Subjects provided blood at 48, 72 , and $96 \mathrm{~h}$ after a 12 - to 14 -h fasting period, whereas the 32 - and 56-h blood samples were taken four hours after participants had consumed a light, low-fat snack. Participants remained in the hospital only for the first $24 \mathrm{~h}$ of the turnover study. They 
returned when necessary for subsequent blood sampling. Subjects were provided with the AHA phase 1 isocaloric diet during their stay in the hospital and were provided thereafter with a similar three-meal-per-day outpatient diet that they could consume at home or at work.

Isolation of HDL and apo A-I for radioactivity counting. Blood was sampled in Vacutainer tubes containing sodium EDTA, and plasma was immediately separated from red cells by centrifuging at $2,000 \mathrm{~g}$ for $20 \mathrm{~min}$ at $4^{\circ} \mathrm{C}$. HDL was isolated by sequential ultracentrifugation as already described here. Aliquots of total plasma and of the $d<1.063 \mathrm{~g} / \mathrm{ml} \mathrm{HDL}$ and $d>1.21 \mathrm{~g} / \mathrm{ml}$ plasma fractions were taken to measure the proportion of radioactivity found in each of these fractions. To measure the radioactivity specifically associated with apo A-I, $100-\mu \mathrm{l}$ aliquots of the dialyzed HDL were delipidated as described previously (15) and dissolved overnight in a $0.5 \mathrm{M}$ phosphate buffer with $1 \%$ SDS, $1 \%$ mercaptoethanol ( $\mathrm{pH}$ 7.2). The samples were then run on $15 \%$ SDS-PAGE to isolate apo A-I. The apolipoproteins were stained overnight with R-250 Coomassie blue colloidal stain and destained for two hours in water, and the apo A-I band was sliced from the gel. Radioactivity in each plasma fraction and in the apo A-I band on the gel was counted in a Beckman 5500 gamma counter (Beckman Instruments Inc.). The apo A-I radioactivity in the gel was taken as a percentage of the radioactivity found in that gel and adjusted to total HDL radioactivity in counts per minute per milliliter. The recovery of radioactivity from the SDS gels compared with that applied to the gel ranged from $70 \%$ to $85 \%$. All radioactivity counts were corrected for the half-life of the isotope and adjusted back to the time of injection.

Fractionation of HDL by immunoaffinity chromatography. We developed a method that allowed the separation of LPAI and LpAI:AII in small quantities with a high degree of reproducibility. A mixture of commercially available polyclonal antibody for apo A-II (catalog no. 726486; Boehringer Mannheim GmbH Diagnostica, Montreal, Quebec, Canada) was coupled to CNBr-activated Sepharose 4B (Pharmacia Biotech AB, Uppsala, Sweden) as instructed by the manufacturer $(5-10 \mathrm{mg} / \mathrm{g}$ gel). Before use, the immunosorbent was washed with an equal volume of a $0.15 \mathrm{M} \mathrm{NaCl}, 0.01 \mathrm{M}$ EDTA, $0.05 \mathrm{M}$ Tris-HCl, 0.01 $\mathrm{M} \mathrm{NaN}_{3}$ buffer ( $\mathrm{pH} 7.4$ ), hereafter referred to as chromatography buffer, by gentle agitation for five minutes followed by a short spin at $500 \mathrm{~g}$. Aliquots of $0.5 \mathrm{ml}$ of the anti-apo A-II immunosorbent was added to $1.5-\mathrm{ml}$ Eppendorf tubes for each sample to chromatograph. The gel in the Eppendorf tubes was allowed to pack for $20 \mathrm{~min}$. The remaining buffer was discarded while verifying that all tubes contained the same quantity of immunosorbent. A standard of apo A-II labeled with ${ }^{125}$ I was run in parallel for each set of analyses to provide an assessment of the capacity of the immunosorbent to adsorb apo A-II. The radioactivity in LpAI and in LpAI:AII was determined by first adding $25 \mu \mathrm{l}$ of the isolated HDL fraction to the immunosorbent along with $475 \mu \mathrm{l}$ of the chromatography buffer. The mixture was then agitated gently on a vortex shaker with an Eppendorf attachment for two hours, after which the gel was allowed to pack for $20 \mathrm{~min}$. The supernatant containing the unbound HDL particles (LPAI) was removed, and the anti-apo A-II immunosorbent was washed a second time by adding $500 \mu \mathrm{l}$ of the chromatography buffer and by gentle agitation for one hour. The supernatant containing unbound LPAI was removed after the gel had been allowed to pack for $20 \mathrm{~min}$. Fractions containing the unbound LPAI from the first and second extractions were mixed and counted for radioactivity. The immunosorbent containing the bound LpAI:AII lipoproteins was removed from the Eppendorf tube with buffer and counted for radioactivity. The total recovery of radioactivity using this micromethod for the separation of LpAI and LPAI:AII was greater than $90 \%$ of that applied to the immunosorbent. The nonretained fraction of the ${ }^{125}$ I-labeled apo A-II standard usually contained less than $5 \%$ of the radioactivity applied to the gel. The coefficient of variation of the method, measured using a standard of radiolabeled apo A-II on consecutive gels and sample replicates in preliminary experiments, was $5.8 \%$.

Determination of HDL particle size. HDL size was measured by nondenaturing 4-30\% PAGE (gels purchased from David Rainwater, Southwest Foundation for Biomedical Research, San Antonio, Texas, USA) as described previously (16). Peak and mean radii of small and large HDL particles were determined by densitometric scanning (ImageMaster DTS densitometer with ImageMaster computer software; Pharmacia Biotech AB) based on relative migration distance of standard-molecularweight proteins of known diameter (HMW Calibration Kit; Pharmacia Biotech Inc., Piscataway, New Jersey, USA). The estimated radius of the major peak in each scan was identified as the HDL peak particle size. A weighted (or mean) HDL particle size was also calculated using a modification of the method described by Li et al. (22). The mean HDL particle size for each subject was obtained by multiplying the size of each band by its fractional area. This mean HDL particle radius (in nanometers) therefore combines the HDL size distribution as well as the relative concentration of each HDL band (22).

Laboratory measurements. Cholesterol was measured using the CHOD-PAP enzymatic colorimetric kit (Boehringer Mannheim GmbH Diagnostica). Protein was measured by the technique described by Lowry et al. (23). Triglycerides were measured as esterified glycerol using an enzymatic colorimetric kit (Boehringer Mannheim GmbH Diagnostica). Free glycerol was eliminated from the sample in a preliminary reaction followed by enzymatic hydrolysis of TG, with subsequent determination of the liberated glycerol by colorimetry. Phospholipids were measured using a kit from Boehringer Mannheim GmbH Diagnostica (catalog no. 691844). Apo A-I and apo A-II were measured in total plasma and in the various HDL fractions by electroimmunoassay.

As already discussed here, the phospholipid and apolipoprotein content of fasting and TG-enriched HDL was characterized further in a subgroup of six additional study participants who had been subjected to the five-hour Intralipid infusion protocol. The phospholipid content of HDL before and after Intralipid was analyzed according to standardized procedures $(24,25)$. Briefly, HDL samples were extracted with chloroform $/$ methanol $(2: 1 \mathrm{vol} / \mathrm{vol})$. After partitioning with added saline, the lower phase was passed through a small column of anhydrous sodium sulfate and taken to dryness under nitrogen. The sample was dissolved in chloroform and then subjected to normal-phase HPLC using a Spherisorb $3-\mu \mathrm{m}$ column (inside diameter: $100 \times 4.6-\mathrm{mm}$; Alltech Associates, Deerfield, Illinois, USA) installed into a Hewlett-Packard Model 1060 liquid chromatograph (Hewlett-Packard, Palo Alto, California, USA). The column was connected to a Hewlett-Packard Model 5988B quadrupole mass spectrometer equipped with a nebulizer-assisted electrospray ionization interface. Positive-ion spectra were taken in the mass range 350-1,100. The molecular species of the various phospholipids were identified on the basis of the molecular mass provided by the mass spectrometer and the knowledge of the fatty acid composition of phospholipid classes and the relative chromatographic retention time (26). The apo E (27) and apo C-III (28) content of fasting and TG-enriched HDL in the second subset of six study participants was quantified by ELISA as described previously. HDL apo C-I concentrations were also measured by ELISA using anti-human polyclonal antibodies.

Kinetic analysis. The radioactivity die-away curves were analyzed using a two-pool model as described previously $(15,16)$. This model assumes the existence of an intravascular pool in equilibrium with a nonvascular pool. All losses from the system 
Table 1

Participants' individual plasma lipid profile

\begin{tabular}{lcccc}
\hline Subject & $\begin{array}{c}\text { Cholesterol } \\
\mathrm{mmol} / \mathrm{l}\end{array}$ & $\begin{array}{c}\text { Triglycerides } \\
\mathrm{mmol} / \mathrm{l}\end{array}$ & $\begin{array}{c}\mathrm{HDL}-\mathrm{C} \\
\mathrm{mmol} / \mathrm{l}\end{array}$ & $\begin{array}{c}\text { Total/HDL-C } \\
\text { ratio }\end{array}$ \\
1 & 2.8 & 0.69 & 1.07 & 2.6 \\
2 & 4.4 & 0.88 & 1.11 & 4.0 \\
3 & 3.4 & 0.84 & 0.94 & 3.6 \\
4 & 4.6 & 0.87 & 0.95 & 4.8 \\
5 & 4.0 & 0.80 & 1.05 & 3.8 \\
6 & 2.5 & 0.68 & 0.90 & 2.8 \\
Mean \pm SEM & $3.6 \pm 0.4$ & $0.79 \pm 0.04$ & $1.00 \pm 0.04$ & $3.6 \pm 0.3$ \\
& & & & \\
\hline
\end{tabular}

C, cholesterol.

are assumed to occur from the intravascular pool only. The FCR of apo A-I in fasting and TG-enriched HDL was derived directly from the kinetic model as the clearance rate from the intravascular pool, using the SAAM II software (SAAM Institute, Seattle, Washington, USA). Parameter estimates were obtained for each individual and tracer, and mean parameter values were determined. The HDL apo A-I production rate was derived using the FCR of apo A-I from fasting HDL particles, multiplied by the HDL apo A-I pool size and by plasma volume (estimated using individual's weight $\times 0.045$ ).

The two-pool model could adequately describe the kinetics of the radiolabeled apo A-I in the total HDL fraction. Indeed, individual estimation of apo A-I FCR in fasting or TG-enriched HDL using this model was generally associated with low coefficients of variation $(<15 \%)$, a criterion that reflects the degreeof-fit of a model to data (this value is calculated by dividing the SD associated with the estimation of a kinetic parameter by SAAM-II with the estimated parameter). Apo A-I radioactivity data in the various HDL subfractions were analyzed using absolute values (in counts per minute per milliliter) as well as specific activities (counts per minute per milligram). The radioactivity die-away curves for LpAI and LpAI:AII were also analyzed using the two-pool model, although the fit of the model to the data slightly decreased, probably because further fractionation of the HDL fraction introduced more "noise" into the data, thereby reducing the fit of a given model.

Statistics. Results are presented as mean \pm SEM. One-way ANOVA for repeated measures was used to test differences in FCR and composition between fasting and TG-enriched HDL in the various HDL subfractions. Correlation analysis was per-

Table 2

Mean lipid and protein characterization of the fasting and TG-enriched HDL tracers

\begin{tabular}{lcccc}
\hline & Fasting HDL & TG-rich HDL & Difference $(\%)^{\mathrm{A}}$ & $P$ value $^{\mathrm{B}}$ \\
& \multicolumn{2}{c}{$\mathrm{mg} / \mathrm{dl}$} \\
Triglyceride & $9.0 \pm 0.9$ & $18.9 \pm 2.4$ & 111.2 & \\
Cholesterol & $38.8 \pm 1.4$ & $41.7 \pm 2.9$ & 7.9 & 0.002 \\
Esters & $32.3 \pm 2.4$ & $34.1 \pm 3.6$ & 5.6 & 0.19 \\
Free & $6.4 \pm 1.3$ & $7.6 \pm 1.5$ & 34.2 & 0.12 \\
Phospholipids & $61.6 \pm 3.7$ & $76.2 \pm 5.6$ & 25.0 & 0.03 \\
Proteins & $184.2 \pm 15.4$ & $190.0 \pm 19.2$ & 4.1 & 0.38 \\
Apo A-I & $146.1 \pm 8.4$ & $159.2 \pm 17.7$ & 9.2 & 0.22 \\
Apo A-II & $17.9 \pm 3.5$ & $16.3 \pm 2.4$ & -5.1 & 0.32 \\
HDL size & \multicolumn{2}{c}{ Radius (nm) } & & \\
Mean & $4.56 \pm 0.10$ & $4.60 \pm 0.08$ & 1.3 & 0.68 \\
Peak & $4.49 \pm 0.08$ & $4.64 \pm 0.21$ & 3.3 & 0.52
\end{tabular}

Values are mean \pm SEM. These concentrations were measured on the cold HDL tracers (before radioiodination). Very similar numbers were obtained when analyzing the composition of the radiolabeled HDL. ${ }^{A}$ Mean within-subject relative difference between TG-enriched HDL and fasting HDL. ${ }^{B}$ Obtained by one-way ANOVA for repeated measures. formed using the Pearson and Spearman correlation coefficients for parametric and nonparametric variables, respectively. Analyses were performed using the Statistical Analysis System (SAS Institute, Cary, North Carolina, USA).

\section{Results}

Plasma lipoprotein, lipid, and apolipoprotein concentrations, and lipoprotein sizes presented in this report are the mean of four fasting determinations made during the course of the HDL turnover study. All participants were normolipidemic for their age (Table 1$)$. The physiological variation in plasma TG, cholesterol, apo AI, and HDL cholesterol, calculated using the concentrations measured at four different time points during the turnover study, averaged $22 \%, 5 \%, 11 \%$, and $9 \%$, respectively. Thus, metabolic variables and body weight were stable throughout the course of the HDL turnover study (results not shown), indicating that the studies were performed under steady-state conditions.

Table 2 presents the mean lipid, protein, and apolipoprotein composition of the fasting and TGenriched HDL tracers. The average TG content of HDL increased 2.1-fold (from $9.0 \pm 0.9$ to $18.9 \pm 2.4 \mathrm{mg} / \mathrm{dl} ; P=$ $0.002)$. The five-hour infusion of Intralipid also resulted in a significant $25 \%$ increase in total phospholipid $(P=$ 0.03 ), whereas total cholesterol, total protein, apo A-I, and apo A-II levels were not affected to a significant extent. The ratio of phospholipids to protein also did not change significantly with Intralipid (not shown). Consistent with this observation is the fact that Intralipid had no effect on HDL peak or mean particle size.

Figure 1 presents the relative proportion of phosphatidylcholine, sphingomyelin, and lysophosphatidylcholine in fasting and TG-rich HDL particles. These results were obtained from the second sample of six normolipidemic individuals subjected to the Intralipid infusion protocol only (see Methods). Intralipid resulted in a significant reduction in the proportion of sphingomyelin $(P=0.02)$, the least abundant phospholipid subclass, whereas relative levels of phosphatidylcholine and lysophosphatidylcholine did not change significantly with Intralipid. As shown in Table 3, TG enrichment of HDL with Intralipid resulted in a 54\% reduction in apo $\mathrm{C}$-III $(P=0.07)$, whereas reductions in apo $\mathrm{E}$ $(-33.7 \%)$ and apo C-I $(-26.3 \%)$ did not reach statistical significance. Apo A-I was also not modified with Intralipid in this second sample of individuals, thus confirming results from the original sample of six study participants (results not shown).

The majority (74-93\%; mean of $84 \%$ ) of the injected radioactivity was recovered during the turnover studies within the HDL fraction, and there was no difference in the amount of radioactivity recovered in HDL between fasting HDL and TG-enriched HDL (not shown). The proportion of plasma radioactivity in the $d<1.063 \mathrm{~g} / \mathrm{ml}$ fraction ranged from $2.2 \%$ to $9.7 \%$ and was similar whether derived from the fasting or the TG-enriched HDL (5.7 \pm $1.1 \%$ vs. $6.7 \pm 0.9 \%$, respectively; $P=0.42$ ). The proportion of total plasma radioactivity in the $d>1.21 \mathrm{~g} / \mathrm{ml}$ fraction derived from the TG-enriched HDL was also similar to that derived from fasting HDL $(10.5 \pm 0.8 \%$ vs. $9.5 \pm 2.1 \%$, respectively; $P=0.72$ ). Variations in the proportion of 


\section{Figure 1}

Relative proportion, in percent, of phosphatidylcholine (PC), sphingomyelin (SM), and lysophosphatidylcholine (LPC) in fasting and TG-enriched HDL tracers. Values are expressed as a percentage of all three phospholipid subclasses. This analysis was performed on a second sample of six normolipidemic subjects to characterize further the phospholipid content of HDL with Intralipid infusion (see Methods). The plasma lipid profile of these six subjects was similar to that of the six individuals who participated in the HDL turnover study.

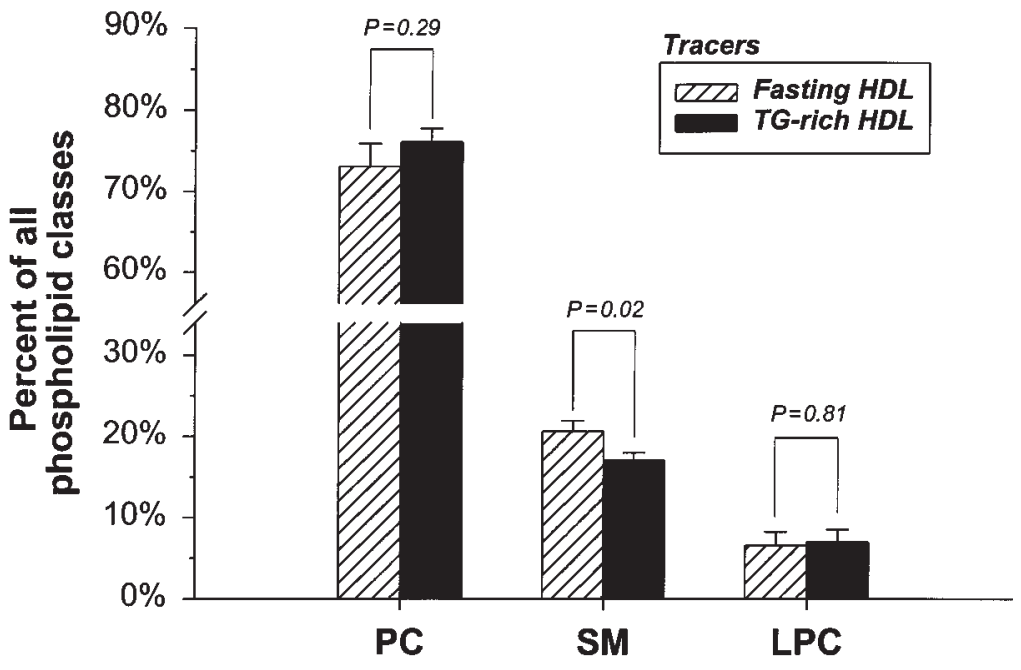

radioactivity in these fractions during the turnover studies were minimal, and thus, the kinetics of the non-HDL fractions could not provide additional information on the kinetics of HDL in relation to TG enrichment.

TG enrichment of HDL and apo A-I catabolism. The FCR derived from the die-away curves of radiolabeled HDL apo A-I from fasting and TG-enriched HDL are presented in Table 4, and the mean HDL apo A-I clearance curves for each tracer are illustrated in Figure 2. Apo A-I from TG-enriched HDL was cleared more rapidly than fasting HDL apo A-I in five of six experiments. As a result, TG enrichment of HDL increased the FCR of apo A-I by an average of $26 \%(0.025 \pm 0.010$ vs. $0.020 \pm$ $0.009 / \mathrm{h})$ compared with fasting HDL $(P=0.02)$. As shown in Figure 2, the increased catabolic rate of HDL apo A-I associated with TG-enriched HDL compared with fasting HDL was apparent even in the early stages of the turnover study (from five hours to one day), and the divergence of the radioactivity die-away curves between fasting and TG-enriched HDL was accentuated over a longer period (two to five days).

In one individual, the FCRs of TG-enriched and fasting HDL apo A-I were virtually identical (subject no. 5). The Intralipid infusion in subject no. 5 yielded the smallest increase in the TG content of HDL $(11.5 \mathrm{mg} / \mathrm{dl}$ for fasting HDL; $18.4 \mathrm{mg} / \mathrm{dl}$ for TG-enriched HDL). Figure 3 depicts the correlation between the Intralipid-induced increase in the TG content of HDL and the magnitude of the increase in HDL apo A-I FCR seen with TG enrichment of HDL. This highly significant correlation $(r=$ $0.97, P=0.001)$ also emphasizes the close relationship between the TG content of HDL and apo A-I catabolism. Intralipid-induced changes in other constituents of HDL (phospholipids, free or esterified cholesterol, apoproteins) showed no significant relationship with the Intralipid-induced increase in apo A-I FCR ( $r$ ranging from -0.36 to $0.14 ; P>0.5$ ).

Effect of TG enrichment of HDL on the FCR of LPAI and LPAI:AII. The individual and mean kinetic data for LPAI and LPAI:AII subfractions derived from each tracer are presented in Table 5. The FCR of TG-enriched LPAI particles was systematically more rapid compared with fast- ing LpAI in all five experiments. Thus, the mean FCR of TG-enriched LpAI $(0.028 \pm 0.005 / h)$ was $32.2 \%$ greater than that derived from fasting LPAI $(0.022 \pm 0.006 / \mathrm{h} ; P$ $<0.05)$. On the other hand, there was no systematic difference in the clearance of fasting and TG-enriched LpAI:AII $(F C R=0.042 \pm 0.019$ vs. $0.030 \pm 0.006 / h$, respectively; $P=0.43)$. Exclusion of one participant from the analysis (subject no. 3) with a very high FCR of fasting LPAI:AII (FCR $=0.117 / \mathrm{h})$ had essentially no impact on the results. The FCR of LPAI particles was significantly correlated with the TG content of the injected tracers $(r$ $=0.81, P=0.005)$, whereas FCR of LPAI:AII showed no significant association with the TG content of HDL $(r=$ $-0.33, P=0.36)$. These results suggest that the enhanced clearance of HDL apo AI from HDL enriched with TG is largely accounted for by differences in clearance of LpAI but not LPAI:AII.

\section{Discussion}

TG enrichment of HDL and apo A-I catabolism. To our knowledge, this study provides the first direct evidence in humans that enrichment of HDL with TG enhances the metabolic clearance of apo A-I from the circulation. A twofold increase in the TG content of HDL, resulting from the Intralipid infusion, was associated with a significant $26 \%$ increase in the FCR of HDL apo A-I. This effect of TG enrichment of HDL on the catabolism of apo A-I was seen in five of six participants. The Intralipid-induced TG enrichment of HDL

Table 3

Apo E, C-I, and C-III in fasting and TG-enriched HDL tracers Fasting HDL TG-rich HDL Difference (\%) ${ }^{\mathrm{A}} \quad P$ value $^{\mathrm{B}}$

$\mathrm{mg} / \mathrm{dl}$

Apo E Apo C-1 Apo C-III

$$
\begin{aligned}
& 4.8 \pm 0.7 \\
& 4.6 \pm 0.6
\end{aligned}
$$$$
4.5 \pm 0.6
$$

$$
\begin{aligned}
& 3.2 \pm 1.0 \\
& 3.4 \pm 0.6 \\
& 2.0 \pm 0.7
\end{aligned}
$$

$-33.7$

$-26.3$

0.32

Values are mean \pm SEM. These measurements were performed in the second sample of six normolipidemic individuals subjected to a five-hour Intralipid infusion. These subjects did not participate in the HDL apo A-I kinetic studies. AMean within-subject relative difference between TG-enriched HDL and fasting HDL. ${ }^{B}$ Obtained by one-way ANOVA for repeated measures. 


\section{Figure 2}

Mean die-away curves of apo A-I radioactivity from fasting and TGenriched HDL in six human participants. The data presented on the $y$ axis represent the radioactivity on HDL apo A-I isolated by gel electrophoresis of the delipidated HDL fraction (see Methods). The radioactivity data for each tracer were first expressed as a specific activity and then normalized as a function of the radioactivity measured at the first time interval (10 min). The curves were generated by fitting a two-pool model (twoexponential function) to the data. Values are mean \pm SEM.

was significant but not massive, making the present findings as physiologically relevant as possible. It must be emphasized that the TG content of both fasting and postprandial HDL particles of hypertriglyceridemic individuals is usually far greater than the TG enrichment of HDL achieved with Intralipid in the normotriglyceridemic subjects of the present study $(29,30)$.

Although the Intralipid-induced changes in the TG content of HDL represented the most important and significant change among all HDL components, parallel changes in phospholipids (25\%) and cholesterol (7.9\%) were noted. Intralipid contains significant amounts of phospholipids (particularly phosphatidylcholine) and cholesterol. Although the total phospholipid mass in TG-enriched HDL was increased by $25 \%$ compared with that in fasting HDL, the relative proportion of the two major subclasses of phospholipids (phosphatidylcholine and lysophosphatidylcholine), which represented more than $90 \%$ of total HDL phospholipids, was not modified to a significant extent by Intralipid (Figure 1). On the other hand, the proportion of sphingomyelin decreased slightly but significantly with Intralipid. In light of the data suggesting that variations in the phospholipid content of HDL may affect its charge and, hence, its stability (31), future studies will have to determine whether these small changes may have affected HDL apo A-I catabolism to a significant degree.

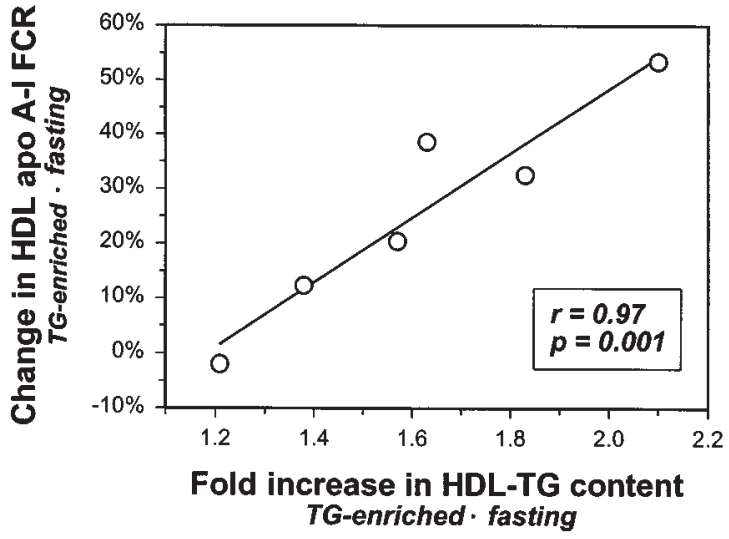

Figure 3

Correlation between the Intralipid-induced -fold increase in the TG content of HDL and the simultaneous increase in HDL apo A-I FCR. A similar correlation with the increased HDL apo A-I FCR was observed when changes in the TG content of HDL after the five-hour Intralipid infusion were expressed as changes in percent rather than -fold increase $(r=0.80$, $P=0.05$; not shown). The correlation was obtained using the -fold increase in the TG content of HDL of the radiolabeled (injected) tracer.

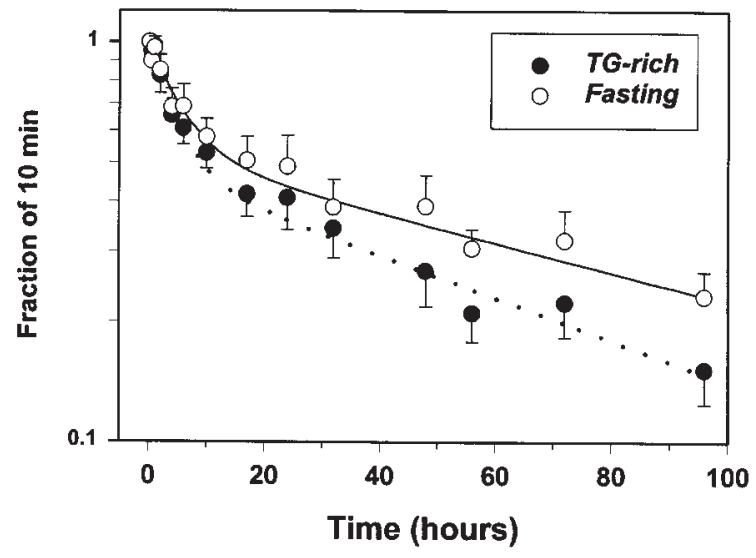

Infusion of Intralipid also resulted in significant reductions of apo E, apo C-III, and apo C-I, but not of apo A-I and apo A-II. Reduction in the apo E content of HDL would not appear as one of the mechanisms through which TG enrichment of HDL enhances the clearance of apo A-I, as it is generally assumed that apo E mediates the uptake of lipoproteins (32). On the other hand, Le et al. (33) have reported that apo C-III was inversely correlated with HDL apo A-I FCR. It is therefore possible that part of the enhanced clearance of apo A-I from TG-enriched HDL may be attributed to simultaneous changes in apo C-III. However, there was a very strong and significant correlation between the Intralipid-induced -fold increase in the TG content of HDL and the simultaneous increase in apo A-I catabolism ( $r=0.97$; Figure 3$)$, despite the limited sample size. On the other hand, no other change in the constituents of HDL showed such a strong association with changes in HDL apo A-I catabolism. These observations reinforce the importance of TG enrichment of HDL in determining its metabolic fate.

We have not attempted to use an oral fat load as a means to enrich HDL with TG. However, in light of the fact that the changes in HDL composition seen with Intralipid infusion were very similar to those that are generally observed after a fatty meal (29), we anticipate that enriching HDL with TG through an oral fat meal may give rise to similar results. This hypothesis, however, remains to be specifically tested.

TG enrichment and catabolism of LpAI and LPAI:AII particles. Previous reports have emphasized the differential kinetics of LPAI and LpAI:AII particles (34), and results of the present study tend to support this concept. The turnover rate of apo A-I in LpAI particles has been shown to be substantially faster than that of apo A-I on LPAI:AII particles (34). Urine/plasma radioactivity data from the same study also suggested that the faster catabolism of apo A-I on LpAI was not primarily due to exchange with LpAI:AII particles or with extravascular compartments, but rather, to irrevocable elimination and degradation (34). The present study was not primarily designed to compare the clearance of LpAI and LpAI:AII. In addition, we have not specifically examined the clearance of A-I on LPAI or LPAI:AII, but rather, have studied the clearance of all apolipoproteins in LpAI and LpAI:AII particles. However, apo A-I accounts for more than $70 \%$ of the proteins in HDL and even a greater proportion in LPAI particles (35). We found 
that TG enrichment of HDL systematically enhanced the clearance of LpAI in all subjects by an average of $32 \%$. In fact, most of the increase in HDL apo A-I clearance due to TG enrichment of HDL appeared to be related to an increase in LpAI catabolism but not in LpAI:AII. In concordance with these observations was the fact that the TG content of the HDL tracers (fasting and TG-rich) correlated significantly with the FCR of LPAI but not with the FCR of LPAI:AII. It must be stressed, however, that the reliability of the model to describe the kinetics of LPAI:AII was limited because of relatively low radioactivity counts and the relatively high noise in the data, thus reducing our ability to detect a significant difference. Therefore, a significant but somehow less-marked contribution of LPAI:AII particles on the enhanced clearance of apo A-I from TG-enriched HDL cannot be ruled out with confidence based on the present data. Our analysis of the effect of TG enrichment on LpAI and LpAI:AII metabolism was not the primary hypothesis tested, and additional studies will have to be undertaken to determine clearly whether TG enrichment of HDL has a different impact on the metabolic clearance of LpAI and LpAI:AII particles.

Is apo A-I loosely bound to TG-enriched HDL? A number of hypotheses can be put forth to explain the increased catabolism of apo A-I on TG-enriched HDL. In a landmark paper, Horowitz et al. (13) compared the kinetics of apo A-I on HDL that had been whole-labeled and HDL that had been labeled by exchange with homologous radiolabeled apo A-I (exchange-labeled HDL) in normotriglyceridemic and hypertriglyceridemic individuals. The authors reported that the proportion of apo A-I dissociating from the exchange-labeled HDL was much greater than that of whole-labeled HDL in all subjects. They also reported that individuals with reduced plasma HDL cholesterol levels who also had increased plasma TG levels had a greater proportion of their apo A-I in an easily dissociable form. These conclusions were drawn from the observation that the $d>1.21 \mathrm{~g} / \mathrm{ml}$ plasma fraction contained more labeled and unlabeled apo A-I upon ultracentrifugation. In the present study, there was no apparent difference between TG-enriched and fasting HDL in the proportion of radioactivity found in the $d>1.21 \mathrm{~g} / \mathrm{ml}$ fraction during the turnover studies. Fasting and TGenriched HDL in the present study were whole-labeled. In addition, TG-enriched HDL obtained by infusing Intralipid for five hours was isolated by ultracentrifugation and thus was subjected to two centrifugations at $d=$ $1.21 \mathrm{~g} / \mathrm{ml}$ before reinjection. It may be argued that any loosely bound apo A-I resulting from the TG enrichment of HDL could have fallen off the particle during the isolation procedures. It is therefore not surprising that the proportion of label in the $d>1.21 \mathrm{~g} / \mathrm{ml}$ fraction during the turnover study was similar between the two tracers. Although apo A-I may bind with varying affinity to HDL particles, this phenomenon does not appear to explain the differences in the FCR of apo A-I between fasting and TGenriched HDL that were observed in the present study.

Importance of lipolytic modification of HDL. Several enzymes play important roles in the metabolism of HDL by modifying its composition and size. Hepatic lipase is one of the most important lipolytic enzymes involved in HDL metabolism (36). Hepatic lipase promotes the for-
Table 4

FCR of apo A-I from fasting and TG-rich HDL

\begin{tabular}{lccccc}
\hline Subject & \multicolumn{5}{c}{ FCR $(\mathrm{CV}, \%)^{\mathrm{A}}$} \\
& Fasting & HDL & TG-rich & HDL & $(\%)^{\mathrm{B}}$ \\
1 & 0.012 & $(6.7)$ & 0.017 & $(7.5)$ & 38.6 \\
2 & 0.018 & $(5.5)$ & 0.024 & $(5.6)$ & 32.5 \\
3 & 0.038 & $(5.5)$ & 0.043 & $(3.7)$ & 12.3 \\
4 & 0.018 & $(14.8)$ & 0.028 & $(5.2)$ & 53.4 \\
5 & 0.021 & $(8.3)$ & 0.021 & $(4.4)$ & -2.0 \\
6 & 0.014 & $(3.0)$ & 0.016 & $(3.2)$ & 20.4 \\
Mean \pm SEM & $0.020 \pm 0.009$ & $0.025 \pm 0.010$ & $25.9{ }^{\mathrm{C}}$
\end{tabular}

AThe coefficient of variation (CV), provided in parentheses, is the SD associated with the estimation of the FCR by SAAM II divided by the FCR value, expressed in percent. The CV is an estimate of the degree-of-fit of the model to the data. The lower the CV, the better the fit of the model to the data. ${ }^{\mathrm{B} T h e}$ relative increase $\left(^{+}\right)$ or reduction (-) in the clearance of TG-rich HDL when compared with fasting HDL particles. ${ }^{C}$ Significant difference $(P=0.02)$ between fasting and TG-rich HDL.

mation of small HDL by cleaving triglycerides and phospholipids from larger HDL particles (37). It has been shown that TG-enriched HDL is a better substrate for hepatic lipase than TG-poor HDL $(11,12)$. The impact of hepatic lipase on TG-enriched HDL is therefore significant in hypertriglyceridemic states and may explain to a large extent the presence of smaller and denser HDL particles in these states (38). CETP, another enzyme that plays a crucial role in HDL metabolism, is indirectly involved in this process (10). CETP is responsible for the transfer of neutral lipids in the circulation, particularly by favoring the transfer of TG and cholesteryl esters between TG-enriched lipoproteins and HDL. In hypertriglyceridemic states, there is an increased transfer of TG from TG-enriched lipoprotein to HDL and of cholesteryl ester from HDL to TG-enriched lipoproteins via the action of CETP. This process ultimately results in the TG enrichment of HDL (10). A study in CETP-transgenic mice has shown that CETP expression leads to TG enrichment of HDL, making it more vulnerable to lipol-

\section{Table 5}

Mean ${ }^{A}$ FCR of LpAI and LpAI:All from fasting and TG-rich HDL

\begin{tabular}{lrrrrr}
\hline & \multicolumn{5}{c}{ FCR $(\mathrm{CV}, \%)^{\mathrm{A}}$} \\
Subject & Fasting HDL & TG-rich & HDL & $\begin{array}{c}\text { Difference } \\
\text { LpAl particles }\end{array}$ \\
2 & & & & & \\
3 & 0.011 & $(18.3)$ & 0.016 & $(6.4)$ & 50.5 \\
4 & 0.014 & $(27.2)$ & 0.018 & $(30.7)$ & 24.3 \\
5 & 0.035 & $(7.4)$ & 0.040 & $(7.8)$ & 13.6 \\
6 & 0.015 & $(12.6)$ & 0.025 & $(9.2)$ & 64.1 \\
Mean \pm SEM & 0.036 & $(5.2)$ & 0.039 & $(9.0)$ & 8.4 \\
LpAl:All particles & $0.022 \pm 0.006$ & $0.028 \pm 0.005$ & 32.2 \\
2 & & & & & \\
3 & 0.016 & $(52.1)$ & 0.023 & $(51.7)$ & 43.2 \\
4 & 0.117 & $(11.2)$ & 0.049 & $(8.3)$ & -58.3 \\
5 & 0.023 & $(12.8)$ & 0.029 & $(9.0)$ & 24.6 \\
6 & 0.030 & $(7.5)$ & 0.033 & $(7.0)$ & 10.2 \\
Mean \pm SEM & 0.023 & $(15.0)$ & 0.014 & $(15.0)$ & -40.4 \\
& $0.042 \pm 0.019$ & $0.030 \pm 0.006$ & -4.1
\end{tabular}

Measurements were not performed in study participant no. 1. AThe coefficient of variation $(\mathrm{CV})$, provided in parentheses, equals the SD derived from the estimation of the FCR by SAAM II divided by the estimated FCR, expressed in percent. BThe relative increase $(+)$ or reduction $(-)$ in the clearance of TG-rich HDL when compared with fasting $\mathrm{HDL}$ particles. ${ }^{C}$ Significantly different $(P=0.01)$ from fasting HDL. 
ysis by circulating lipase, and causing apo A-I to be shed from the particles (18). Horowitz et al. (13) reported similar findings. Indeed, increased renal clearance of HDL apo A-I when infused through rabbit kidneys occurred only when TG-rich HDLs were previously subjected to lipolysis by hepatic lipase and lipoprotein lipase. We have also reported that, in rabbits, small, lipolytically modified human HDL particles were cleared more rapidly from the circulation than large, TG-rich human HDL (15). More recently, we reported that TG enrichment of $\mathrm{HDL}$, in the absence of prior in vivo lipolytic modification of the particle, had no impact on the rate at which small and large HDL paticles are cleared in rabbits (16).

Intralipid infusion had no significant impact on HDL particle size in the present study. This lack of size change may be due to a number of factors. The duration of TG enrichment of the particles with Intralipid may have been insufficient to result in a significant reduction in HDL size by lipolysis (i.e., possibly sustained and repeated TG enrichment of HDL is required for HDL size reduction). It must also be stressed that the twofold increase in the TG content of HDL was reached after five hours of Intralipid infusion. The magnitude of TG enrichment from zero to three to four hours may not have been enough to generate smaller particles through nonstimulated intravascular lipase activity. Finally, Intralipid contained a significant amount of cholesterol that may have been transferred to HDL, which may have prevented reduction in particle size with TG loading.

We assume that the TG-enriched HDL may have been hydrolyzed to some extent by hepatic lipase during the kinetic study. This process is responsible for the generation of small, lipolytically modified HDL particles that appear to have an enhanced catabolism (15). The possibility that there may have been a precursor-product relationship within TG-rich HDL (large particles being converted to small HDL through lipolysis) was not investigated in the present study. Nevertheless, we speculate that the increased susceptibility of TG-rich HDL to intravascular lipolysis by hepatic lipase and other circulating lipases, compared with fasting HDL, may account for the enhanced catabolism of TG-enriched HDL apo A-I.

A second important function for both lipoprotein lipase and hepatic lipase has recently been described, in addition to their role in the hydrolysis of TG in lipoproteins. Indeed, it appears that both lipases can mediate the binding and uptake of lipoproteins into cells (11). Thus, studies in hepatic lipase-transfected hepatocytes have shown that hepatic lipase and apo E may utilize similar heparan sulfate proteoglycan binding sites to mediate HDL uptake (39). The relative importance of cell-surface binding sites in the overall metabolism of HDL, and whether this mechanism is involved in the increased clearance of TGenriched HDL, remains to be determined.

The HDL turnover studies in this report were performed over a five-day period, whereas most of the previous HDL turnover studies have been performed over a longer period, usually 12 to 14 days. Shorter studies may overestimate "true" FCR values (40). As expected, the mean residence time of apo A-I on fasting HDL calculated from the FCR obtained in the present study averaged 2.3 days, a value lower than the four- to five-day plasma residence time that has been reported elsewhere $(34,41,42)$. The accuracy of describing the kinetics of apo A-I from fasting HDL and TG-enriched HDL based on this two-pool model was, however, acceptable, as reflected by the low coefficients of variation associated with the estimation of HDL apo A-I FCR. Data presented in Figure 2 also clearly show that there is sufficient information from 20 to $96 \mathrm{~h}$ to derive the slope of the second exponential of the radioactivity die-away curve. It must be stressed that our study was not designed to provide true FCR values for apo A-I in fasting and TGenriched HDL, but rather, to compare the clearance of these particles. Because the FCRs of apo A-I on fasting and TG-enriched HDL were compared simultaneously in the same individual, underestimation of the true kinetic values for each tracer is likely to have been similar for each tracer. The use of a longer study protocol may have had the undesirable effect of diluting any actual difference in clearance between the two tracers because of exchange of apolipoproteins among plasma lipoproteins. In fact, a longer study protocol may have attenuated the difference between fasting and TG-rich HDL. To the extent that exchange of labeled apolipoproteins occurred in the present study, the fact that we were able to distinguish the catabolism of the two HDL tracers implies that the physiological difference between the FCR of apo A-I on TG-enriched and fasting HDL may be even greater than the differences derived from the analysis of the kinetic curves. Finally, a difference in the clearance of TG-rich and fasting particles was apparent even in the early part of the kinetic study (Figure 2). For those reasons, we are confident that the five-day protocol we have selected to use has enabled us to test definitively the hypothesis that TG enrichment of HDL enhances the metabolic clearance of HDL apo A-I in humans.

To our knowledge, this study provides the first direct evidence in humans that TG enrichment of HDL is associated with a significant increase in the rate at which HDL apo A-I is cleared from the circulation. It must be emphasized that the impact of TG enrichment of HDL on its catabolism may be seen even in the presence of small, physiologically relevant increases in the TG content of HDL, such as those observed after a fatty meal. The clinical implications of the present findings are also important. Indeed, it may be hypothesized that small reductions in the TG content of HDL, such as those achieved with TG-lowering agents, exercise, or weight loss, may have a significant beneficial impact on the metabolism of HDL particles in the circulation. Variations in the TG content of HDL in humans may have fundamental implications for the regulation of apo A-I, and consequently, for determining plasma HDL cholesterol concentrations in humans.

\section{Acknowledgments}

We are greatly indebted to Pat Harley for her nursing assistance. We also thank the research volunteers for participating in the study. The contribution of Arnis Kuksis in analyzing the phospholipid content of HDL is also fully acknowledged. This work was supported by an Operating Grant from the Heart and Stroke Foundation of Ontario (to G.F. Lewis). B. Lamarche is a Research Scholar of the Medical Research Council of Canada. 
A. Carpentier is a recipient of a research fellowship from the Juvenile Diabetes Foundation. G.F Lewis is a Career Investigator of the Heart and Stroke Foundation of Canada. J.S. Cohn is supported by an operating grant from the Medical Research of Canada (MT-14684). P.H.R. Barrett is supported by National Institutes of Health grants RR-12609 and HL-49110.

1. Miller, G.J., and Miller, N.E. 1977. Plasma high density lipoprotein concentration and development of ischaemic heart disease. Lancet. 1:16-18.

2. Durrington, P.N. 1993. How HDL protects against atheroma. Lancet. 342:1315-1316.

3. Reichl, D., and Miller, N.E. 1989. Pathophysiology of reverse cholesterol transport. Insight from inherited disorders of lipoprotein metabolism. Arterioscler. Thromb. Vasc. Biol. 9:785-797.

4. Lamarche, B., et al. 1993. Metabolic heterogeneity associated with high plasma triglyceride or low HDL cholesterol levels in men. Arterioscler. Thromb. 13:33-40.

5. Brinton, E.A., Eisenberg, S., and Breslow, J.L. 1991. Increased apo A-I and apo A-II fractional catabolic rate in patients with low high density lipoprotein-cholesterol levels with or without hypertriglyceridemia. J. Clin. Invest. 87:536-544.

6. Nestel, P.J. 1987. High-density lipoproteins turnover. Am. Heart J. 113:518-521.

7. Brinton, E.A., Eisenberg, S., and Breslow, J.L. 1994. Human HDL cholesterol levels are determined by apoA-I fractional catabolic rate, which correlates inversely with estimates of HDL particle size. Effects of gender, hepatic and lipoprotein lipases, triglyceride and insulin levels, and body fat distribution. Arterioscler. Thromb. 14:707-720.

8. Hayek, T., et al. 1993. Hypertriglyceridemia and cholesteryl ester transfer protein interact to dramatically alter high density lipoprotein levels, particle sizes, and metabolism. Studies in transgenic mice. J. Clin. Invest. 92:1143-1152.

9. Rao, S.N., Magill, P.J., Miller, N.E., and Lewis, B. 1980. Plasma high-density lipoprotein metabolism in subjects with primary hypertriglyceridaemia: altered metabolism of apoproteins AI and AII. Clin. Sci. 59:359-367.

10. Tall, A.R. 1995. Plasma cholesteryl ester transfer protein and high-density lipoproteins: new insights from molecular genetic studies. J. Intern. Med. 237:5-12.

11. Beisiegel, U. 1996. New aspects on the role of plasma lipases in lipoprotein catabolism and atherosclerosis. Atherosclerosis. 124:1-8.

12. Olivecrona, G., and Olivecrona, T. 1995. Triglyceride lipases and atherosclerosis. Curr. Opin. Lipidol. 6:291-305.

13. Horowitz, B.S., et al. 1993. Increased plasma and renal clearance of an exchangeable pool of apolipoprotein A-I in subjects with low levels of high density lipoprotein cholesterol. J. Clin. Invest. 91:1743-1752.

14. Clay, M.A., Newnham, H.H., and Barter, P.J. 1991. Hepatic lipase promotes a loss of apolipoprotein A-I from triglyceride-enriched human high density lipoproteins during incubation in vitro. Arterioscler. Thromb. 11:415-422.

15. Lewis, G.F., et al. 1997. Clearance of post-prandial and lipolytically modified human HDL in rabbits and rats. J. Lipid Res. 38:1771-1781.

16. Lamarche, B., Uffelman, K.D., Barrett, H.R., Steiner, G., and Lewis, G.F. 1998. Analysis of particle size and lipid composition as determinants of the metabolic clearance of human high density lipoproteins in a rabbit model. J. Lipid Res. 39:1162-1172.

17. Hayek, T., et al. 1993. Hypertriglyceridemia and cholesteryl ester transfer protein interact to dramatically alter high density lipoprotein levels, particles sizes, and metabolism. J. Clin. Invest. 92:1143-1152.

18. Melchior, G.W., et al. 1994. Apolipoprotein A-I metabolism in cholesteryl ester transfer protein transgenic mice. Insights into the mechanisms responsible for low plasma high density lipoprotein levels. J. Biol. Chem. 269:8044-8051.

19. Clay, M.A., Newnham, H.H., Forte, T.M., and Barter, P.J. 1992. Cholesteryl ester transfer protein and hepatic lipase activity promote shedding of apo A-I from HDL and subsequent formation of discoidal HDL. Biochim. Biophys. Acta. 1124:52-58.

20. Goldberg, I.J., Blaner, W.S., Vanni, T.M., Moukides, M., and Ramakrish- nan, R. 1990. Role of lipoprotein lipase in the regulation of high density lipoprotein apolipoprotein metabolism. Studies in normal and lipoprotein lipase-inhibited monkeys. J. Clin. Invest. 86:463-473.

21. Mcfarlane, A.S. 1958. Efficient trace labeling of proteins with iodine. Nature. 182:53-57.

22. Li, Z., McNamara, J.R., Ordovas, J.M., and Schaefer, E.J. 1994. Analysis of high density lipoproteins by a modified gradient gel electrophoresis method. J. Lipid Res. 35:1698-1711.

23. Lowry, O.H., Rosebrough, N.J., Farr, A.L., and Randall, R.J. 1951. Protein measurement with the Folin phenol reagent. J. Biol. Chem. 193:265-275.

24. Myher, J.J., and Kuksis, A. 1984. Determination of plasma total lipid profiles by capillary gas-liquid chromatography. J. Biochem. Biophys. Methods. 10:13-23.

25. Kuksis, A., Myher, J.J., Marai, L., and Geher, K. 1975. Determination of plasma lipid profiles by automated gas chromatography and computerized data analysis. J. Chromatogr. Sci. 13:423-430.

26. Pruzanski, W., et al. 1998. Lipoproteins are substrates for human secretory group IIA phospholipase A2: preferential hydrolysis of acute phase HDL. J. Lipid Res. 39:2150-2160.

27. Cohn, J.S., et al. 1996. Plasma concentration of apolipoprotein $\mathrm{E}$ in intermediate-sized remnant-like lipoproteins in normolipidemic and hyperlipidemic subjects. Arterioscler. Thromb. Vasc. Biol. 16:149-159.

28. Fredenrich, A., et al. 1997. Plasma lipoprotein distribution of apoC-III in normolipidemic and hypertriglyceridemic subjects: comparison of the apoC-III to apoE ratio in different lipoprotein fractions. J. Lipid Res. 38:1421-1432.

29. Lewis, G.F., et al. 1990. Postprandial lipoprotein metabolism in normal and obese subjects: comparison after the vitamin A fat-loading test. $J$. Clin. Endocrinol. Metab. 71:1041-1050.

30. Lewis, G.F., et al. 1991. Fasting hypertriglyceridemia in non-insulindependent diabetes mellitus (NIDDM) is an important predictor of postprandial lipid and lipoprotein abnormalties. J. Clin. Endocrinol. 72:934-944.

31. Davidson, W.S., Sparks, D.L., Lund-Katz, S., and Phillips, M.C. 1994. The molecular basis for the difference in charge between pre-beta- and alphamigrating high density lipoproteins. J. Biol. Chem. 269:8959-8965.

32. Miller, N.E. 1990. HDL metabolism and its role in lipid transport. Eur. Heart J. 11(Suppl. H):1-3

33. Le, N.A., Gibson, J.C., and Ginsberg, H.N. 1988. Independent regulation of plasma apolipoprotein C-II and C-III concentrations in very low density and high density lipoproteins: implications for the regulation of the catabolism of these lipoproteins. J. Lipid Res. 29:669-677.

34. Rader, D.J., Castro, G., Zech, L.A., Fruchart, J.C., and Brewer, H.B., Jr. 1991. In vivo metabolism of apolipoprotein A-I on high density lipoprotein particles LpA-I and LpA-I,A-II. J. Lipid Res. 32:1849-1859.

35. Skinner, E.R. 1994. High-density lipoprotein subclasses. Curr. Opin. Lipidol. 5:241-247.

36. Eisenberg, S. 1984. High density lipoprotein metabolism. J. Lipid Res. 25:1017-1058.

37. Zhong, S., et al. 1994. Human apoA-II inhibits the hydrolysis of HDL triglyceride and the decrease of HDL size induced by hypertriglyceridemia and cholesteryl ester transfer protein in transgenic mice. J. Clin. Invest. 94:2457-2467.

38. Murakami, T., et al. 1995. Triglycerides are major determinants of cholesterol esterification/transfer and HDL remodeling in human plasma. Arterioscler. Thromb. Vasc. Biol. 15:1819-1828.

39. Ji, Z.S., Dichek, H.L., Miranda, R.D., and Mahley, R.W. 1997. Heparan sulfate proteoglycans participate in hepatic lipase and apolipoprotein Emediated binding and uptake of plasma lipoproteins, including high density lipoproteins. J. Biol. Chem. 272:31285-31292.

40. Champarnaud, G., Ouguerram, K., Magot, T., and Lutton, C. 1992. Overestimation of the lipoprotein fractional catabolic rate (FCR) measured in short duration experiments. J. Pharmacobiodyn. 15:541-546.

41. Vega, G.L., Gylling, H., Nichols, A.V., and Grundy, S.M. 1991. Evaluation of a method for study of kinetics of autologous apolipoprotein A-I. J. Lipid Res. 32:867-875.

42. Schaefer, E.J., et al. 1982. Human apolipoprotein A-I and A-II metabolism. J. Lipid Res. 23:850-862. 